\title{
Infectious Diseases of Poverty reviewer acknowledgement 2015
}

Xiao-Nong Zhou

\section{Contributing reviewers}

The editors of Infectious Diseases of Poverty would like to thank all our reviewers who have contributed to the journal in volume 4 (2015).

Hanin Abdel-Haq
Italy

Suneth Agampodi

Sri Lanka

Fiacre A.R. Agossa

France

Valéria Magalhães Aguiar

Brazil

Maryam Alavi

Australia

Nahid Ali

India

Shahzad Ali

Pakistan

Julio Aliberti

United States of America

Salem Alkoshi

Malaysia

Jaffar Al-Tawfiq

Saudi Arabia

Frederico Figueiredo Amâncio

Brazil

Fentie Ambaw

United Kingdom

Stephen Ambu

Malaysia
Zuhair Amr

Jordan

SS Andreadis

Greece

Andrea Angheben

Italy

Gloria Ansa

Ghana

Maria Antoniou

Greece

Ahmed Arshad

Pakistan

G. V. Asokan

Bahrain

Justine Assenga

Tanzania, United Republic Of

Salah Awaidy

Oman

James Ayukepi Ayukekbong

Sweden

Santi P Sinha Babu

India

Subash Babu

India

David Barbosa

Brazil
Sukhadeo Barbuddhe

India

Amy Barrette

Switzerland

Devra Barter

United States of America

Leonardo Basco

France

Vagner Wilian Batista

Brazil

Soeren Becker

Germany

\section{Tatiana Belkina}

Czech Republic

Yunus E. Beyhan

Turkey

Andrea K Blanchard

Canada

David Blok

Netherlands

Boakye Boatin

Ghana

Monika Bociaga-Jasik

Poland

Maha Bouzid

United Kingdom

Correspondence: xiaonongzhou1962@gmail.com

National Institute of parasitic Diseases, Shanghai, China CDC, China 
Barry M. Bradford

United Kingdom

\section{Andreia F Brilhante}

Brazil

Christine Budke

United States of America

Jesse Bump

United States of America

Stephanie Burniston

United Kingdom

Jun Cao

China

Helene Carabin

United States of America

Luís Cardoso

United States of America

Orlando Cenciarelli

Italy

Kwang Poo Chang

United States of America

Himanshu Chaturvedi

India

Jun-Hu Chen

China

Gerardo Chowell

Georgia

Luc Coffeng

Netherlands

Ulisses Confalonieri

Brazil

Ian Gillespie Cook

United Kingdom

James A. Cotton

Canada

Janet Cox-Singh

United Kingdom

Zulma Cucunubá

Colombia

Sabyasachi Das

India

Emmie de Wit

United States of America

Nana Rose Diakite

Cote D'Ivoire
João Carlos Pinto Dias

Brazil

Emilio Dirlikov

Canada

Hui-Fen Dong

China

Yan Du

China

Bereket Duko

Ethiopia

Karthickeyan Duraisamy

India

Valentina Virginia Ebani

Italy

Yehenew Ebstie

Ethiopia

Akinwale Efunshile

Denmark

Grace Egeland

Norway

Uwem Ekpo

Nigeria

Mark Erfe

United States of America

Bruno Eto

France

Shirzad Fallahi

Iran, Islamic Republic Of

Ann Fallon

United States of America

Zhen-Chuan Fan

China

Chia-Kwung Fan

Taiwan, China

Li-Qun Fang

China

Jun Feng

China

Magilé C. Fonseca

Cuba

Florence Fouque

Swaziland

Jana Fried

United Kingdom
Ricardo Fujiwara

Brazil

Francesco Maria Fusco

Italy

Luis-Alberto Gaitán-Cepeda

Mexico

Shu-Jing Gao

China

Amadou Garba

Switzerland

Andrés Garchitorena

United States of America

Melissa N. Garcia

United States of America

Giuliano Garofolo

Italy

Mohammadreza Gholami

Iran, Islamic Republic Of

Luis Miguel González

Spain

Catherine Gordon

Australia

Rajeev Goyal

India

Carlos Graeff-Teixeira

Brazil

Jack ET Grimes

United Kingdom

Gigi Gronvall

United States of America

Ashley Grosso

United States of America

Rodrigo Guabiraba

France

Wei Guo

China

Narayan Gyawali

Australia

Fisaha Haile

Ethiopia

Yara Halasa

United States of America

Nguyen B Hoa

Viet Nam 
Sung-Tae Hong

Korea, Republic Of

Wei Hu

China

Felicien Ilunga-Ilunga

Congo

Asma Iqbal

Canada

Laura F. Jefferys

Germany

Julie Jesson

France

Min-Jun Ji

China

Feng-Liang Jin

China

Maria Vang Johansen

Denmark

Roch Christian Johnson

Benin

Nicholas Johnson

United Kingdom

Seth Judson

United States of America

Mamadou Kaba

South Africa

George Kafatos

United Kingdom

Ajeet Kaushik

United States of America

Faiz Kermani

France

Thomas Kesteman

Madagascar

Samson Kiware

Tanzania

Stefanie Knopp

Switzerland

Ismail Soner Koltas

Turkey

Randall Kramer

United States of America

Stefanie Krauth

Chile
Anuja Krishnan

United States of America

Karen Krogfelt

Denmark

Annette Kuesel

Switzerland

Agnes Kurniawan

Indonesia

Kang Yiu Lai

Hong Kong, China

Moses Laman

Papua New Guinea

Essoya D Landoh

Togo

Samia Laokri

Belgium

David Larsen

United States of America

Anne Laudisoit

United Kingdom

Jae Hoon Lee

Korea, Democratic People'S Republic

Of

Marilena Leis

Italy

Shi-Zhu Li

China

Ying Li

China

Xin-Xu Li

China

Chang-Wei Li

United States of America

Xiang-Rui Li

China

Ie-Bin Lian

Taiwan, China

Marshall Lightowlers

Australia

Wen Lin

China

Ji-Ming Liu

Hong Kong, China
Lu Liu

China

Ming-Yuan Liu

China

Eric S Loker

United States of America

Knut Lönnroth

Switzerland

Anna Lena Lopez

Philippines

Da-Bing Lu

China

Zhao-Rong Lun

China

Shan Lv

China

Ye Ma

China

Ya-Jun Ma

China

Gino C. Matibag

Philippines

Gerald Amandu Matua

Oman

Rooyen Mavenyengwa

Zimbabwe

Webster Mavhu

Zimbabwe

Don McManus

Australia

KE McMillan

Australia

Takafira Mduluza

Zimbabwe

Ann Miller

United States of America

J. Michael Miller

United States of America

Oriol Mitjà

Papua New Guinea

David Molyneux

United Kingdom

Estrella Montero

Spain 
Catrin Moore

United Kingdom

Filbert Mpogoro

Tanzania

Ivo Mueller

Spain

Martin W Mutuku

Kenya

Adamson Muula

Malawi

Ramesh Nagarajappa

India

Pradeep Nair

India

Mercy Nassali

Bangladesh

Ignatius C Ndong

Cameroon

Jintana Ngamvithayapong-Yanai

Thailand

\section{Abel Ntambue}

Congo, The Democratic Republic Of The

\section{Ndudim Ogo}

United States of America

Omoyemi Ogwumike

Nigeria

Olusola Ojurongbe

Nigeria

Anna L. Okello

United Kingdom

Joseli Oliveira-Ferreira

Brazil

Samuel Anu Olowookere

Nigeria

Nyamongo Onkoba

Kenya

Anyebe Onoja

Nigeria

\section{Gabriella Ortore}

Italy

Trevino Pakasi

Indonesia
Jun-Xiong Pang

Singapore

Andrea Pantoja

Swaziland

Daniel M. Parker

Thailand

John Pasick

Canada

Jorge Pedrosa

Portugal

Adalberto Pereira Filho

Brazil

Lucia Monserrat Pérez-Navarro

Mexico

Guey Chuen Perng

Taiwan, China

Punnee Pitisuttithum

Thailand

Ranjan Premaratna

Sri Lanka

Xiao-Peng Qi

China

Men-Bao Qian

China

Zhi-Jiang Qin

China

Soheila Rabiepoor

Iran, Islamic Republic Of

Senaka Rajapakse

Sri Lanka

C.P. Ramachandran

Malaysia

José Manuel Ramos

Ethiopia

Ram Rangsin

Thailand

Felipe Dutra Rêgo

Brazil

Hong-Yan Ren

China

Blanca Restrepo

United States of America

Nicolás Robinson-García

Spain
Minakshi Rohilla

India

Gianluigi Rossi

Italy

Xavier Roura

Brazil

Somenath Roy

India

Nicole Rübsamen

Germany

Charles Rupprecht

United States of America

Maren Johanne Heilskov Rytter

Denmark

Moussa Sacko

Mali

Evans L Sagwa

South Africa

Ashis Saha

India

Rehana Salam

Pakistan

Helena Lúcia Carneiro Santos

Brazil

Ari Winasti Satyagraha

Indonesia

Matthias Schmitz

Germany

Poongulali Selvamuthu India

Emmanuel Serrano

Portugal

Lillian Seu

Zambia

Heba Shawky

Egypt

Yu-Juan Shen

China

Ben-Yun Shi

China

Ambuj Shrivastava

India

Denise Silva

Brazil 
Cheolho Sim

United States of America

Sarah Simmons

United States of America

B.B. Singh

India

Susanne H. Sokolow

United States of America

Maja Stanojevic

Serbia And Montenegro

Michelle Stanton

United Kingdom

Peter Steinmann

Switzerland

Xin-Zhuan Su

United States of America

Xiao-You Su

China

Bindu Sukumaran

Singapore

Irene Sumbele

Cameroon

Claudette Simone Sutherland

Switzerland

Hiroshi Suzuki

Japan

Annika C. Sweetland

Colombia

Katsushi Tajima

Japan

Hiko Tamashiro

Japan

Ernest Tambo

South Africa

Chee Wah Tan

Malaysia

Biruhalem Taye

Ethiopia

Walter R. J. Taylor

Switzerland

Khin Thet Wai

Myanmar

Roger Thomas

Canada
R.C. Andrew Thompson

Australia

Kamala Thriemer

Belgium

Lucas Tirloni

Brazil

Mohamad-Ali Trad

France

Tiziano Tuccinardi

Italy

Claire M Tully

United Kingdom

Begna Tulu

Ethiopia

Kingsley Ukwaja

Nigeria

Ravi Kant Upadhyay

India

Maria Isabel Veiga

Sweden

Raman Velayudhan

Switzerland

Patrícia Sampaio Tavares

Brazil

Elvina Viennet

Australia

Maria Vitale

Italy

Tyson Volkmann

United States of America

Dominique Angèle Vuitton

France

Anjuli Wagner

United States of America

Wei Wang

China

Yong Wang

China

Shan-Qing Wang

China

Ming-Gui Wang

China

Qin Wang

China
Kinley Wangdi

Australia

David Weetman

United Kingdom

Xiao-Lin Wei

China

Mitchell Weiss

Switzerland

Thomas Weitzel

Germany

Yu-Feng Wen

China

Timothy Eoin West

United States of America

Bruce Wilcox

Thailand

La'Marcus Wingate

United States of America

Viroj Wiwanitkit

Thailand

Wendy Wong

Hong Kong, China

Shou-Li Wu

China

Wolfgang Kratzer

Germany

Shang Xia

China

Ning Xiao

China

Jian-Nong Xu

United States of America

Jing $\mathrm{Xu}$

China

Kuang-Yao Yang

Taiwan, China

Kun Yang

China

Guo-Jing Yang

China

Peiling Yap

Switzerland

Hosup Yoon

Singapore 
Chee-Fu Yung

Singapore

Houria Zait

Algeria

Zai-Xing Zhang

China

Hao-Bing Zhang

China

Long-Xian Zhang

China
Yi Zhang

China

Shao-Sen Zhang

China

Jin-Kou Zhao

Switzerland

Xiao-Nong Zhou

China

Xia Zhou

China
Yi-Biao Zhou

China

Shui-Sen Zhou

China

Denis Zofou

Cameroon

Guan-Yang Zou

China 